Correspondence: Vera Auchynka, Republican Scientific and Practical Centre for Pulmonology and Tuberculosis, 157, Dolginovsky trakt, 220053 Minsk, Belarus.

Tel. +375.29.6521152.

E-mail: veraavchinko@mail.ru

Key words: New anti-tuberculosis drugs; fluoroquinolone resistance; rifampicin resistance; second-line injectable drug resistance; adverse drug safety monitoring.

Authors' contributions: All the authors contributed to the conception of the study and protocol development. VA, SS, data collection and data entry; YS, AK, VA, DK, data analysis and interpretation; VA, AK, DK, first draft of the paper. All the authors reviewed the paper critically and gave final approval of the paper.

Conflict of interest: None declared

Funding: This study was funded by the United States Agency for International Development. The funding body had no role in study design, data collection and analysis, decision to publish, or preparation of the manuscript. We used the existing resources in the National Tuberculosis Programme to carry out the data collection.

Ethics: Permission to access the data was obtained from the National Tuberculosis Programme in Belarus. Ethics approval was obtained from the Ethics Committee of the Republican Scientific and Practical Centre for Pulmonology and Tuberculosis in Belarus. Exemption from an ethics review was also received from the World Health Organization Research Ethics Review Committee based in Geneva, Switzerland (ERCefcarddelcontreg/17.03.2020). A waiver of informed consent was granted by ethics review bodies, as the study collected and analysed deidentified routine recording and reporting data.

Disclaimer: The authors alone are responsible for the views expressed in this publication and they do not necessarily represent the decisions or policies of the World Health Organization.

Acknowledgements: The authors thank the national TB counterparts of the Republican Scientific and Practical Centre for Pulmonology and Tuberculosis (RSPCPT) of Belarus for defining research questions and providing data for this study, and the secretariat of the European TB Research Initiative (ERI-TB) at the WHO Regional Office for Europe for organizing the Structured Operational Research Training (SORT-TB) for eastern European countries supported by the USAID-WHO regional partnership project to End TB in eastern Europe. SORT-TB curriculum was an adaptation of the UNICEF/UNDP/World Bank/WHO Special Programme for Research and Training in Tropical Diseases (TDR) SORT IT course (https://www.who.int/tdr/capacity/strengthening/sort/en/) to the eastern European context.

Received for publication: 10 July 2020.

Accepted for publication: 31 July 2020.

${ }^{\circ}$ World Health Organization 2021.

All rights reserved. The WHO Regional Office for Europe has granted the Publisher permission for the reproduction of this article.

Licensee PAGEPress, Italy

Monaldi Archives for Chest Disease 2021; 91:1647

doi: 10.4081/monaldi.2021.1647

Open access statement: In accordance with WHO's open-access publication policy for all work funded by WHO or authored/co-authored by WHO staff members, the WHO retains the copyright of this publication through a Creative Commons Attribution IGO licence (http://creativecommons.org/licenses/by/3.0/igo/legalcode) which permits unrestricted use, distribution and reproduction in any medium provided the original work is properly cited.

\section{Effectiveness and cardiovascular safety of delamanid-containing regimens in adults with multidrug- resistant or extensively drug-resistant tuberculosis: A nationwide cohort study from Belarus, 2016-18}

\author{
Vera Auchynka ${ }^{1}$, Ajay M.V. Kumar, ${ }^{2,3,4}$, \\ Hennadz Hurevich ${ }^{1}$, Yuliia Sereda ${ }^{5}$, \\ Varvara Solodovnikova ${ }^{1}$, Dzmitry Katovich ${ }^{6}$, \\ Svetlana Setkina $^{7}$, Askar Yedilbayev ${ }^{5}$, \\ Aliaksandr Skrahin ${ }^{8}$, Alena Skrahina ${ }^{1}$ \\ ${ }^{1}$ Republican Scientific and Practical Centre for \\ Pulmonology and Tuberculosis, Minsk, Belarus; \\ ${ }^{2}$ International Union Against Tuberculosis and Lung \\ Disease, Paris, France; ${ }^{3}$ International Union Against \\ Tuberculosis and Lung Disease, South-East Asia Office, \\ New Delhi, India; ${ }^{4}$ Yenepoya Medical College, Yenepoya \\ (Deemed to be University), Mangaluru, India; ${ }^{5}$ World \\ Health Organization, Regional Office for Europe, \\ Copenhagen, Denmark; ${ }^{\circ}$ Pulmonary Centre Klinikum \\ Mittelbaden GmbH, Baden-Baden, Germany; ${ }^{7}$ Center for \\ Examination and Tests in Health Service, Minsk, \\ Belarus; ${ }^{8}$ Belarusian State Medical University, Minsk, \\ Belarus
}


mild and managed easily. The most common cardiovascular AEs were QTcF prolongation (64/177, 36\%) and other electrocardiography (ECG) abnormalities $(40 / 177,23 \%)$. There were two instances of serious AEs leading to death, both of which were not related to delamanid. In multivariable analysis, male sex (aHR 0.72; 95\% CI 0.51-0.99), and baseline ECG abnormalities (aHR $1.68 ; 95 \%$ CI 1.19-2.36) were associated with cardiovascular AEs. Median time to culture conversion was 1.1 months (interquartile range: 1.0-2.1). Culture conversion was observed in $115(92 \%)$ patients at six months of treatment and 110 (88\%) completed the treatment successfully. Loss to follow-up, failure and death were observed in $6 \%, 4 \%$ and $2 \%$ patients respectively. Among those assessed at 12 months post-treatment $(n=33)$, recurrence was seen in one patient. The only factor associated with unsuccessful treatment outcomes in multivariable analysis was baseline Hepatitis C co-infection (aHR 3.61; 95\% CI 1.09-11.95). In conclusion, treatment using delamanid-containing regimens was effective and had a favourable safety profile. We hope our findings inform the development of national clinical guidelines and scale-up of new drugs in other countries.

\section{Introduction}

Tuberculosis (TB) still remains a major public health concern worldwide, even though the causal agent was discovered more than 130 years ago. The World Health Organization (WHO) estimated that ten million people developed TB in 2018 and about $15 \%$ of them died due to it. This makes TB the leading infectious cause of death globally, ranking above HIV/AIDS [1]. The global community has pledged to end the TB epidemic by 2030 in line with the sustainable development goals of the United Nations [2]. The progress towards achieving this goal has been slow and varied across the regions. Countries in the WHO European Region are progressing faster with a $5.5 \%$ annual reduction in TB incidence compared to $1.5 \%$ reduction globally [1]. One of the challenges for TB elimination worldwide and especially in the WHO European Region is the epidemic of drug resistant TB.

About half a million people developed multidrug-resistant TB (with resistance to isoniazid and rifampicin) or rifampicin-resistant TB (RR/MDR-TB) in 2018 globally and $6.2 \%$ of them had extensively drug-resistant TB (XDR-TB, with additional resistance to fluoroquinolones and second-line injectable agents). There are enormous gaps in the cascade of care - with nearly two-thirds of estimated M/XDR-TB patients worldwide not diagnosed and/or not started on treatment; of those started on treatment, only half are successfully treated [1]. The poor outcomes are primarily due to lower efficacy, serious adverse drug effects and the long duration of treatment of second line regimens [3].

New TB drugs such as bedaquiline and delamanid have been endorsed by the WHO for management of M/XDR-TB patients and are now available in Belarus for programmatic use [4]. Usage of these drugs has the potential to improve the treatment success rates, reduce the adverse drug events and reduce the duration of treatment [5].

Previous studies on the efficacy and safety of delamanid-containing regimens have shown varying results. A phase III clinical trial among MDR-TB patients showed that there is no difference in culture conversion and treatment success between delamanid and placebo containing regimens $(77.1 \%$ vs $77.6 \%)$ [6,7]. It is not clear whether these results reflect actual drug efficacy or problems in trial design or implementation [8]. There have been many real- world observational studies conducted in different countries on effectiveness of delamanid containing regimens. A report from South Africa in a cohort of difficult-to-treat patients shows high culture conversion [9]. Preliminary findings from the endTB observational cohort study from 17 countries report a $80 \%$ culture conversion during first six months of therapy in cohort of patients treated with delamanid-containing regimens [10]. Meanwhile, there is limited information on final treatment outcomes and there is no information on post-treatment recurrence. A study from South Korea reported a treatment success of $82 \%$ in patients receiving delamanid [11]. There is also limited information about the effect of concomitant use of bedaquiline and delamanid [12,13]. A recent systematic review reports that the combined use of bedaquiline and delamanid had a treatment success of $71.4 \%$ and in only $2.3 \%$ of the patients either drug was stopped due to adverse events [14].

Delamanid has been generally considered safe to use[4]. The most common adverse effect reported was prolongation of QTcF interval, which was mostly mild and manageable at the programmatic level [15]. While about $9-14 \%$ of patients receiving delamanid had QTcF prolongation recorded, in less than 3\% of them, QTcF prolongation exceeded 500 milliseconds [8,16-19]. Yet, information on other cardiovascular adverse events associated with administration of delamanid is limited. It is possible that adverse events are associated with other factors, which include the use of other TB and non-TB medications, antiretrovirals, and baseline clinical and behavioural profile including alcohol and drug use [19]. These factors have not been studied in detail.

Belarus is a country in eastern Europe with a high burden of drug resistant TB. The national TB programme (NTP) in Belarus has been using delamanid since 2016 under programmatic conditions and has had an efficient system of cohort event monitoring including active surveillance and reporting of adverse events [20]. This provides an opportunity to study the effectiveness (including final treatment outcomes and post-treatment recurrence) and cardiovascular safety of delamanid-containing regimen under programmatic settings. This is an important research priority identified by the NTP as well as the regional 'European Tuberculosis Research Initiative' (ERI-TB).

In this study, we aimed to i) describe the sociodemographic and clinical characteristics of adults with M/XDR-TB who received delamanid-containing regimens in Belarus; ii) assess the frequency and incidence of cardiovascular adverse drug events and describe their severity, timing, management and outcome; iii) assess interim and final treatment outcomes and post-treatment recurrence; and iv) assess factors associated with cardiovascular adverse drug events and unsuccessful treatment outcomes.

\section{Materials and Methods}

\section{Study design}

This was a cohort study involving analysis of secondary data routinely collected by the Belarus NTP.

\section{Study population and study period}

We included all adult (aged $\geq 18$ years) patients with $\mathrm{M} / \mathrm{XDR}$ TB who started treatment with delamanid-containing regimens in Belarus from June 2016 to February 2018 at the Republican Scientific and Practical Centre for Pulmonology and Tuberculosis (RSPCPT) and six regional TB hospitals. 


\section{Study setting}

Belarus is a country in eastern Europe with a population of 9.5 million. The Ministry of Health has the overall responsibility for the planning and implementation of the TB Programme in the country. This is done via the RSPCPT based in the capital city Minsk and the health departments of the regions of Belarus.

\section{Diagnosis of MDR-TB and XDR-TB}

The diagnosis and treatment of TB in Belarus follow the national clinical protocol, approved by the Ministry of Health [21]. Patients suspected of TB were tested at regional laboratories or the national reference laboratory located at Republican TB Centre. Appropriate specimens (sputum or any other specimen, including from an extrapulmonary site) were subjected to the following tests: sputum microscopy, Xpert MTB/RIF assay, Line Probe Assay for both first line and second-line drugs and liquid and solid culture and phenotypic drug susceptibility tests (for both first- and second-line drugs on solid and liquid media).

\section{Treatment}

Individualized treatment regimens were designed by the experts in the Republican MDR-TB Concilium taking into account DST results, history of previous treatments and outcomes, co-morbid conditions and clinical status of patient, which was in line with the WHO guidelines and clinical protocols of the NTP $[4,21]$. Treatment regimens were constructed in order to ensure presence of minimum of four effective drugs during the intensive phase. The drugs and dosages are shown in Supplementary Table 1. Treatment consisted of intensive phase for a period of 6-8 months and continuation phase for a period of 12-18 months. Delamanid was usually provided for a period of 24 weeks, but this was extended, if required. Patients were hospitalized for initiation of treatment. Once they become culture negative and clinically stable, patients were discharged from the hospital and referred to a health facility near to their place of residence for continuation of therapy and follow up. Delamanid was administered twice daily and all dosages were directly observed during the entire duration of therapy at inpatient and outpatient settings.

\section{Follow-up and treatment monitoring}

The treatment response was assessed once monthly, by performing sputum culture, microscopy and clinical assessment. Chest radiography is performed once every three months during intensive phase and once every six months during continuation phase. Blood tests are performed every month for assessment of electrolyte abnormalities, albumin levels, anaemia, thrombocytopenia, leukopenia, liver and renal function. Electrocardiography (ECG) is performed once every two weeks in the first month and monthly thereafter for assessment of QTcF interval and detection of arrhythmias.

\section{Grading of adverse events}

The AEs were classified according to the Medical Dictionary for Regulatory Activities (MedDRA) [22]. Grade referred to severity grading scale of adverse events. We used the Common Terminology Criteria for Adverse Events (CTCAE) to grade the adverse events [23]. There were five grades: i) Grade 1 (mild with no or minimal symptoms; requires only careful observation with no intervention); ii) Grade 2 (moderate requiring minimal, local or non-invasive intervention); iii) Grade 3 (medically significant event but not immediately life-threatening; requiring hospitalization or prolongation of hospitalization; disabling; limiting the activities of daily living); iv) Grade 4 (life-threatening conse- quences requiring urgent intervention); v) Grade 5 (death related to adverse event).

\section{Serious adverse event}

AEs are also classified as 'serious AE' or not as defined by The International Council for Harmonisation of Technical Requirements for Pharmaceuticals for Human Use (ICH). As per ICH guideline, a serious adverse event (SAE) is any untoward medical occurrence that at any dose: i) results in death or ii) is life- threatening or iii)requires inpatient hospitalization or results in prolongation of existing hospitalization or iv)result in persistent or significant disability/incapacity or v) results in a congenital anomaly/ birth defect or vi) is a medically important event or reaction [24].

The types of AEs and their grades are shown in Supplementary Table 2. Based on the AE, if the suspected drug is identified, one of the following actions is taken: intensified monitoring, dose reduction, drug stopped temporarily or permanently. Additional supportive treatment is provided, as appropriate.

\section{Recording}

All the patient details are recorded in the case files maintained at the hospitals, the TB treatment cards and the TB register. Case definitions, outcome categories, recording and reporting follow the WHO guidelines [4].

\section{Data variables and sources of data}

The variables related to the study objectives were extracted from the patient charts maintained at the RSPCPT and the regional TB hospitals. These included the sociodemographic, behavioural and clinical data (diagnosis, treatment, adverse events and outcomes). We also collected information on baseline comorbidities including cardiovascular diseases and ECG abnormalities. Culture conversion was defined as obtaining two consecutive culture negative results. Final treatment outcomes were classified as successful (cured and treatment completed) and unsuccessful outcomes (death, loss to follow up, failure, not evaluated).

\section{Data entry and analysis}

The data were double entered and validated in a structured EpiData database created for the purpose of the study. Analysis was done using $\mathrm{R}$ software version 3.5.2 software $\left({ }^{\circ} \mathrm{R}\right.$ Foundation for Statistical Computing, 2016). We described the sociodemographic and clinical profile of study participants by using frequencies and proportions (for categorical variables) and mean (and standard deviation, SD) or median (and interquartile range) for continuous variables, as appropriate. The interim and final treatment outcomes were summarized using frequencies and proportions.

For analysis of AEs, we considered every event as the unit of analysis. The AEs were summarized by type of event, severity and seriousness, time of onset and duration, management and their outcome. The calculation of the incidence rate of SAEs was done by dividing the number of events by the total person-months of observation.

To evaluate associations of sociodemographic and clinical factors with unsuccessful treatment outcomes Cox proportional hazards regression was used. For SAEs, we used an extended Cox regression (The Prentice, Williams, and Peterson Total Time Model) to account for recurrent events. For both outcomes we calculated crude (HR) and adjusted hazard ratios (aHR) and 95\% confidence intervals (CI). Proportionality assumption was tested using by using the Schoenfeld residuals against the transformed time. All variables met the assumption. Variables with $\mathrm{p}$ value $<0.05$ in unadjusted analysis along with 
age and sex were included in the final model. If two variables were collinear, then only one of them was used in the model. We measured collinearity by Variance Inflation Factor. A p value of $<0.05$ was considered as statistically significant in all analyses.

\section{Results}

\section{Patient characteristics}

A total of 125 adult patients were included in the study. Majority of patients were male $(90,72 \%)$ and the mean age (SD) was 43 (12) years. In $44(35 \%)$ of patients, there was a history of alcohol abuse and $79(63 \%)$ were tobacco smokers (Table 1). Most (97\%) of the patients had pulmonary TB and 85 (68\%) had XDR-TB. The following comorbidities were reported at baseline: $\operatorname{HIV}(15 \%)$, hepatitis $\mathrm{C}$ $(15 \%)$, diabetes mellitus (11\%), hypertension (11\%) and cardiovascular disorders (22\%). Nearly $30 \%$ of the patients had baseline ECG abnormalities (non-specific ST segment change, T wave abnormal, overload of the right atrium) with none showing baseline QTcF prolongation (Table 1). All studied patients received delamanid-containing anti-TB treatment, while $20(16 \%)$ patients received both delamanid and bedaquiline. The median duration of delamanid use was 6 months (IQR: 5.5-8.3; range: 1.5-24 months). Nearly half (49\%)

Table 1. Baseline characteristics of M/XDR-TB patients treated with delamanid-containing regimens in Belarus from June 2016 to February $2018(\mathrm{~N}=125)$.

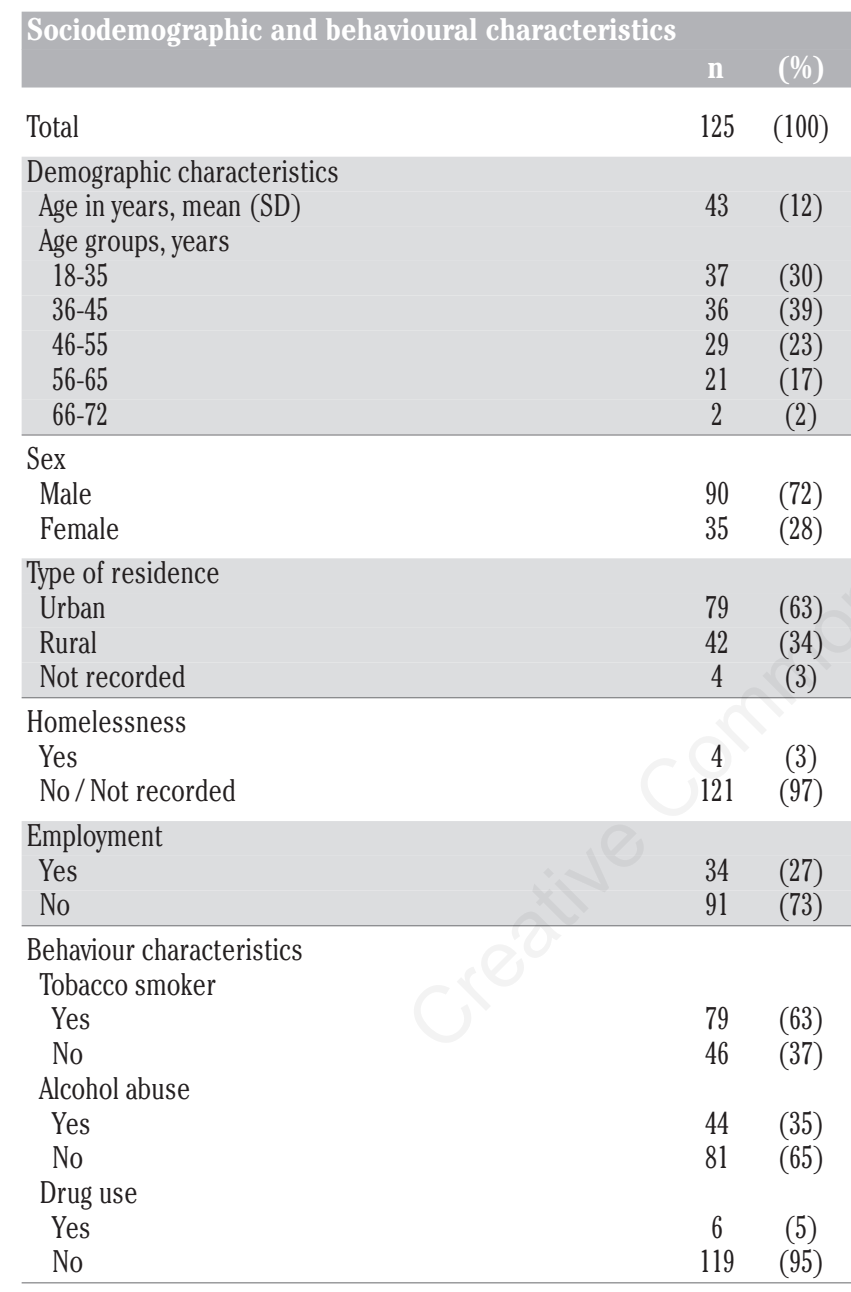

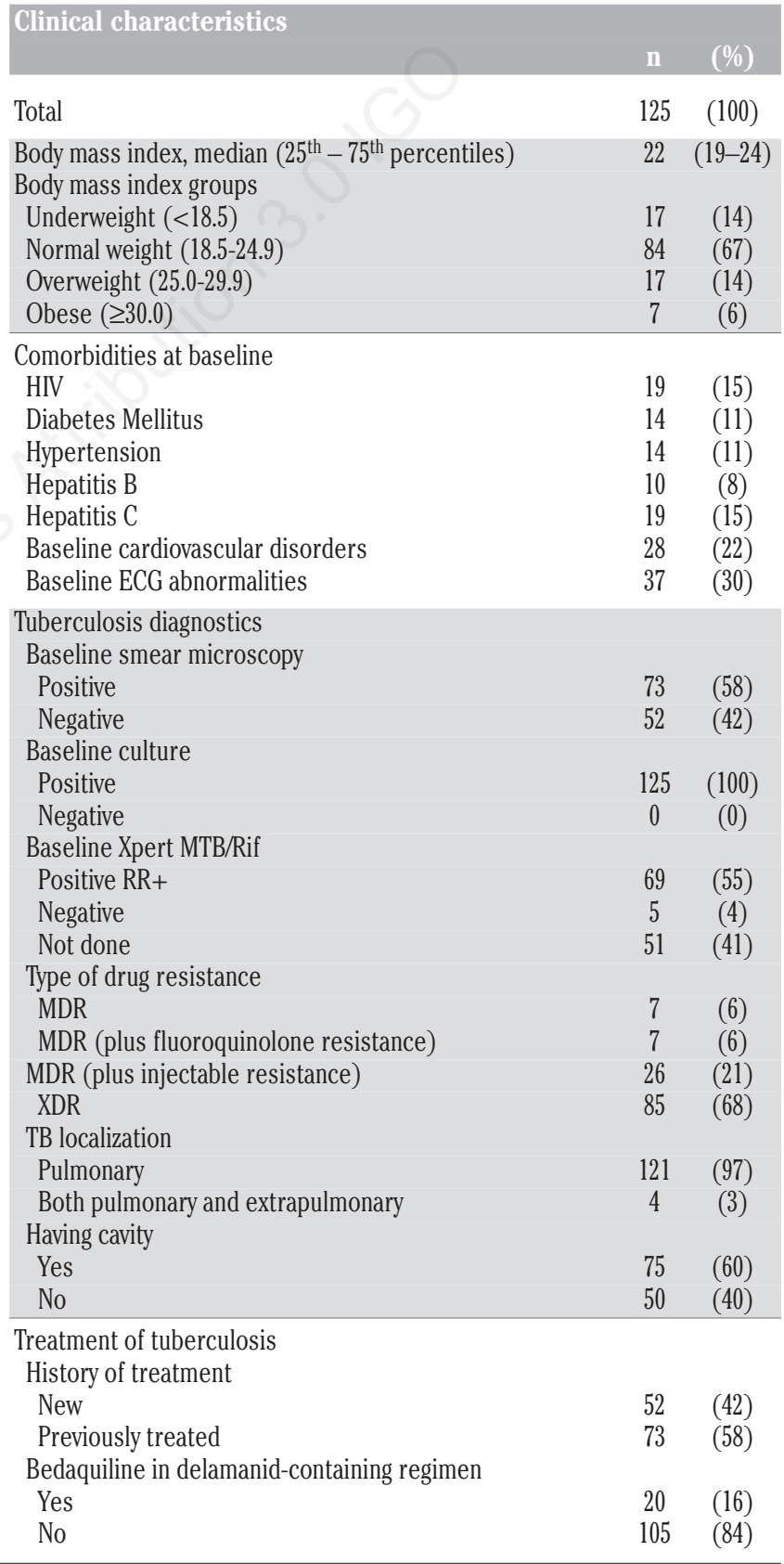


of the patients received delamanid beyond six months of treatment. The median duration of delamanid use was 6 months (IQR: 5.5-8.3 months; Range: 1.5-24 months).

\section{Adverse events}

AEs were reported in all patients. There was a total of 831 AEs recorded. Of these, metabolic and nutritional disorders were the most common (193, 23\%, incidence rate (IR): 7.6 per 100 personmonths) followed closely by the cardiovascular AEs $(177,21 \%$, IR: 7.0 per 100 person-months), renal disorders (107, 13\%, IR: 4.2 per 100 person-months) and blood and lymphatic disorders (106, 13\%, IR: 4.2 per 100 person-months) (Figure 1). The highest incidence of cardiovascular AEs was reported during the first month of treatment which declined steadily as the treatment progressed (Figure 2). The most common cardiovascular AEs were QTcF prolongation $(64 / 177,36 \%)$ and other ECG abnormalities (40/177, $23 \%$ ) (Table 2). In total, 91 of 125 patients (73\%) had cardiovascular AEs, and number of events ranged from 1 to 6 per patient. Most of the AEs were mild in nature and did not require dose adjustment or discontinuation of the drug (temporary or permanent). There were two serious cardiovascular AEs with a fatal outcome that were not related to delamanid therapy. In one patient, death occurred seven months after delamanid was discontinued and the other patient had a severe form of TB meningitis amidst the progression of HIV infection. Most (90\%) of the AEs were resolved by the end of the treatment and the median time to resolve the AEs was 72 (IQR: 31-105) days.

\section{Treatment outcomes}

Culture conversion was observed in $115(92 \%)$ patients at six months of treatment and $110(88 \%)$ completed the treatment successfully (Table 3 ). The median time to culture conversion was 1.1
(IQR: 1.0-2.1; range 0.1-6.9) months. Unsuccessful outcomes were reported in $15(12 \%)$ of patients: $2 \%$ death, $4 \%$ failure and $6 \%$ lost to follow-up. While there was no recurrence at 6 months after treatment, one case $(1 / 33,3 \%)$ of recurrence was recorded after 12 months at post-treatment. There was no statistically significant difference $(p=0.41)$ in treatment success between the delamanid-only cohort $(94 / 105,89 \%)$ and delamanid-plus-bedaquiline cohort (16/20, 80\%).

\section{Factors associated with cardiovascular AEs and unsuccessful outcomes}

In unadjusted analysis, both baseline cardiovascular disorders and baseline ECG abnormalities were associated with increased risk of AEs. Since the two variables were collinear, we included only one of them in multivariable model. In multivariable analysis, male sex (adjusted hazard ratio (aHR) 0.72; 95\% CI 0.51-0.998), and baseline ECG abnormalities (aHR 1.68; 95\% CI 1.19-2.36) were associated with cardiovascular AEs (Supplementary Table 4). Concomitant use of bedaquiline and delamanid was not associated with increased risk of cardiovascular AEs. Hepatitis C co-infection was the only factor associated with unsuccessful treatment outcomes both in unadjusted analysis and multivariable model controlling for sex and age was (aHR 3.61; 95\% CI 1.09-11.95) (Supplementary Table 5).

\section{Discussion}

This is the first study from Belarus reporting on effectiveness and cardiovascular safety of delamanid-containing regimens in adults with RR/MDR-TB and XDR-TB and adds to the global evi-

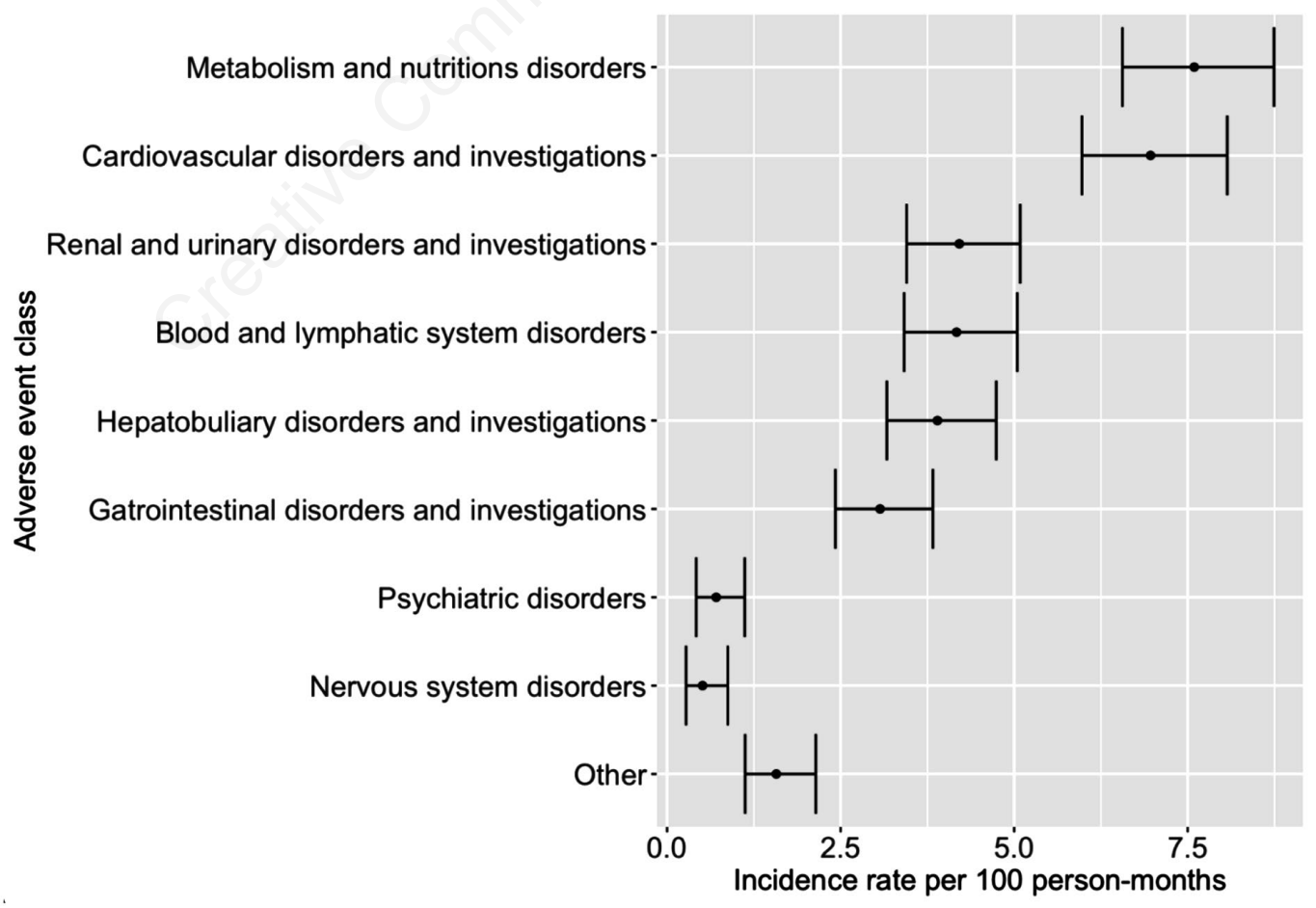

Figure 1. Incidence of adverse events by class among M/XDR-TB patients treated with delamanid-containing regimens in Belarus from June 2016 to February 2018 ( $\mathrm{N}=125)$. MDR, multidrug-resistant; TB, tuberculosis; XDR, extensively drug-resistant. 
dence on this issue. This is the first report globally providing information on post-treatment recurrence. This is also the largest cohort of patients ever reported from programmatic settings.

We found that delamanid-containing regimens were highly effective, safe and well-tolerated among adults with M/XDR-TB in Belarus. The 6-month culture conversion of $92 \%$, treatment success of $88 \%$ and low recurrence post-treatment suggest a high efficacy of the regimen. These are the highest rates of culture conversion and treatment success ever reported in patients with M/XDR-TB. Previous studies reported a 6-month culture conversion ranging 74$84 \%$ and treatment success ranging $61-81 \%[7,10,11,13,25]$.

Cardiovascular AEs have been reported as one of the major

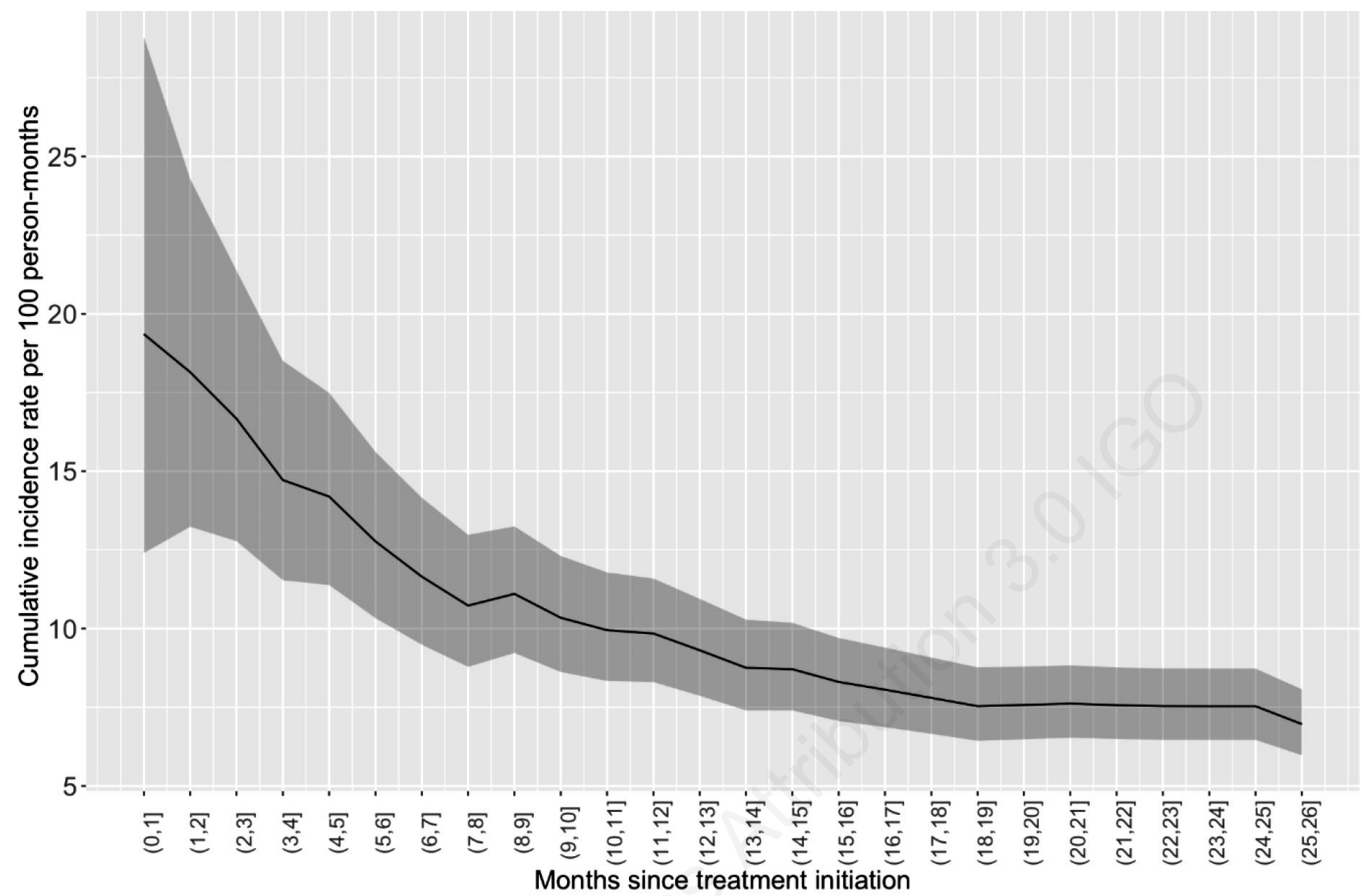

Figure 2. Cumulative incidence rate of cardiovascular adverse drug events along the follow up in M/XDR-TB patients treated with delamanid-containing regimens in Belarus from June 2016 to February $2018(\mathrm{~N}=125)$. Square brackets mean the end point is included, and round parentheses mean it is excluded. For example $(1,2]$ means above the one and below or equal two; MDR, multidrug-resistant; TB, tuberculosis; XDR, extensively drug-resistant.

Table 2. Spectrum of cardiovascular adverse drug events and their severity in M/XDR-TB patients treated with delamanid-containing regimens in Belarus, from June 2016 to February 2018 ( $\mathrm{N}=125)$.

\begin{tabular}{|c|c|c|c|c|c|c|c|c|}
\hline & tion of patients & IR per 100 pm & Number of AE & & & ety & $e^{\#}$ & \\
\hline & $\begin{array}{l}\text { with } \mathrm{AE} \\
\mathrm{n}(\%)^{\circ}\end{array}$ & {$[95 \% \mathrm{CI}]$} & n $(\%) *$ & I & II & III & IV & V \\
\hline QTcF prolongation & $50(40)$ & $2.52[1.94-3.22]$ & $64(36)$ & 40 & 4 & 20 & - & - \\
\hline ECG abnormal & $36 \quad(29)$ & $1.57[1.12-2.14]$ & $40(23)$ & 39 & - & 1 & - & - \\
\hline Sinus tachycardia & $25(20)$ & $1.10[0.73-1.59]$ & $28(16)$ & 28 & - & - & - & - \\
\hline Sinus bradycardia & $19(15)$ & $0.90[0.57-1.36]$ & $23(13)$ & 22 & 1 & - & - & - \\
\hline Ventricular extrasystoles & $5 \quad(4)$ & $0.20[0.06-0.46]$ & $5 \quad(3)$ & - & 4 & 1 & - & - \\
\hline Bundle branch block & $5 \quad(4)$ & $0.20[0.06-0.46]$ & $5 \quad(3)$ & 5 & - & - & - & - \\
\hline Atrioventricular block & $3 \quad(2)$ & $0.12[0.02-0.34]$ & 3 (2) & 2 & 1 & - & - & - \\
\hline Supraventricular extrasystoles & $2 \quad(2)$ & $0.08[0.01-0.28]$ & $2(1)$ & 1 & 1 & - & - & - \\
\hline Hypertension arterial & $2 \quad(2)$ & $0.08[0.01-0.28]$ & $2(1)$ & - & 2 & - & - & - \\
\hline Heart rate irregular & $1 \quad(1)$ & $0.04[0.00-0.22]$ & $1(1)$ & 1 & - & - & - & - \\
\hline Other cardiovascular disorders & $2 \quad(2)$ & $0.08[0.01-0.28]$ & $2(1)$ & - & - & - & - & 2 \\
\hline Other investigations & $2 \quad(2)$ & $0.08[0.01-0.28]$ & $2(1)$ & 2 & - & - & - & - \\
\hline Total & $91(73)$ & $6.96[5.98-8.07]$ & $177(100)$ & 140 & 13 & 22 & - & $2^{\mathrm{d}}$ \\
\hline
\end{tabular}

${ }^{\circ}$ Denominator for the percentage: total sample of the study participants; *denominator for the percentage: total number of respective adverse event occurrences; ${ }^{\sharp}$ grade description: $\mathrm{I}=$ mild, II = moderate, III = severe, IV = life-threatening, V = fatal; AE, adverse events; CI, confidence interval; IR, incidence rate; MDR, multidrug-resistant; QTcF, adjusted QT interval using Fredericia formula; TB, tuberculosis; XDR, extensively drug-resistant; ECG, electrocardiogram. 
concerns when treating with delamanid and bedaquiline. Although nearly three-fourths of patients in our study experienced cardiovascular AEs, most of these were mild and did not require any dose adjustment, discontinuation of drug or treatment. Serious cardiovascular AEs were observed only in two patients and in both cases were not related to delamanid, which is in line with previous studies [17]. The highest incidence of cardiovascular AEs was reported during the first month of the treatment and decreased with the treatment time. This may indicate that there was no cumulative toxicity effect of delamanid-containing regimens related to overall quantity of drug consumed.

Globally and in the WHO European region, the treatment success rates among RR/MDR-TB patients (57\%) and XDR-TB patients $(39 \%)$ have been low and far below the regional target of $75 \%$. The results obtained in this study hold promise that the target of $75 \%$ can be met and exceeded, provided there is access to and rational use of new drugs embedded in a well-performing NTP with an active drug safety monitoring system.

In a sub-group analysis, we found that there was no significant difference in treatment success or risk of cardiovascular AEs between patients who received both bedaquiline and delamanid and those who received delamanid only, although the numbers were small, and we were not adequately powered for this analysis. There have been concerns that using both bedaquiline and delamanid together may act synergistically and increase the risk of cardiotoxicity. Our findings are reassuring because there was no increase in risk of cardiovascular AEs when both drugs were used simultaneously. There were some other interesting findings. Male sex and baseline ECG abnormalities (also baseline cardiovascular disorders) were associated with higher risk of cardiovascular AEs. We do not know the reason for higher risk among males and this needs further study. Nevertheless, these findings indicate that targeting these subgroups for close monitoring during treatment may be helpful. People with hepatitis $\mathrm{C}$ co-infection had a higher risk of unsuccessful TB treatment outcomes. This may be due to multiple reasons including the lack of access to hepatitis $\mathrm{C}$ treatment, possibility of increased hepatotoxicity and poor treatment adherence related to co-existing injectable drug and alcohol use. There is a programme currently in Belarus for diagnosis and management of hepatitis $\mathrm{C}$ patients, but access is challenging due to delays in

Table 3. Treatment outcomes and post-treatment recurrence in M/XDR-TB patients treated with delamanid-containing regimens in Belarus, 06/2016 - 02/2018 ( $\mathrm{N}=125)$.

\begin{tabular}{lcc} 
Characteristics & $\mathrm{n} / \mathrm{N}$ & $(\%)$ \\
Interim treatment outcomes & & \\
$\quad$ Culture negative at 6 months of anti-TB treatment & $115 / 125$ & $(92)$ \\
Final treatment outcomes & & \\
Treatment success & $110 / 125$ & $(88)$ \\
$\quad$ Cured & $110 / 125$ & $(88)$ \\
Treatment completed & $0 / 125$ & $(0)$ \\
Unsuccessful outcome & $15 / 125$ & $(12)$ \\
Treatment failed & $5 / 125$ & $(4)$ \\
Died & $3 / 125$ & $(2)$ \\
Lost to follow-up & $7 / 125$ & $(6)$ \\
\hline Recurrence & & \\
At 6 months & & \\
At 12 months* & $0 / 106$ & $(0)$ \\
\hline
\end{tabular}

${ }^{\circ}$ Among patients who were eligible for 6 -month follow-up assessment after completion of anti-TB treatment ( $\mathrm{N}=106)$; *among patients who were eligible for 12-month follow-up assessment after completion of anti-TB treatment ( $\mathrm{N}=33)$; TB, tuberculosis; MDR, multidrug-resistant; XDR, extensively drug-resistant. diagnosis and treatment. This needs to be addressed to improve the treatment outcomes further.

Our study had several strengths. First, this was a national study with a large number of patients and thus the findings are generalizable to the whole country. Second, Belarus has a comprehensive system of active drug safety monitoring and every AE was identified, graded, recorded (including the dates of appearance and resolution of AEs), reported and managed. This helped in reporting the entire spectrum of cardiovascular AEs in contrast to many other studies which have focused on QTcF interval prolongation only [17]. This also enabled a detailed analysis including calculation of incidence rates, time to development of AEs and time to resolution of AEs.

There were some limitations too. One study limitation relates to the limited availability of delamanid treatment courses during the study period which meant that only about $20 \%$ of all eligible patients received delamanid-containing regimens. In order to prevent development of resistance to delamanid (DST to delamanid was not available during the study period), patients who were more likely to be treatment adherent (assessed based on criteria such as no history of loss to follow-up in previous episodes of TB, availability of a clear address and phone number to enable easy traceability and video observation of treatment during ambulatory phase) were selected by the Republican MDR-TB Consilium. Hence, we cannot rule out a potential selection bias. This might be one reason for the relatively better treatment outcomes reported in the study. We need to wait and watch if the study results will be replicated once delamanid is more widely used for all the eligible patients in the country. Another limitation was that given the small number of events per variable, we could not include more variables in the multivariable model. Finally, we did not have information on recurrence for the entire cohort of patients. We need to follow-up the cohort before making a final conclusion on recurrence rates.

\section{Conclusions}

We found that treatment of adult patients with RR/MDR-TB and XDR-TB patients using delamanid-containing regimens in Belarus was effective and had a favourable cardiovascular safety profile. Nearly nine in ten patients were successfully treated with low rates of post-treatment recurrence. Although the majority of patients experienced cardiovascular AEs, most of them were mild and could be managed easily. Our results provide robust evidence for countries in the WHO European region and elsewhere to introduce and scale-up the use of new drugs.

\section{References}

1. WHO. Global tuberculosis report 2019. Geneva; 2019. Accessed on: 2019 Nov 20. Available from: https://www.who.int/tb/publications/global_report/en/

2. United Nations. Transforming our world: the 2030 agenda for sustainable development transforming our world: the 2030 agenda for sustainable development. 2016.

3. Halleux CM, Falzon D, Merle C, et al. The world health organization global aDSM database: generating evidence on the safety of new treatment regimens for drug-resistant tuberculosis. Eur Respir J 2018;51:1701643.

4. WHO. Consolidated guidelines on drug-resistant tuberculosis 
treatment. Geneva; 2019 Accessed on: 2019 Nov 20. Available from: https://www.ncbi.nlm.nih.gov/books/NBK539517/

5. Migliori GB, Tiberi S, Zumla A, et al. MDR/xdr-tb management of patients and contacts: challenges facing the new decade. the 2020 clinical update by the global tuberculosis network. Int J Infect Dis 2020;92:S15-25.

6. WHO. Position statement on the use of delamanid for multidrug-resistant tuberculosis. Geneva; 2018 Accessed on: 2019 Nov 22. Available from: https://www.who.int/tb/publications/ 2018/WHOPositionStatementDelamanidUse.pdf?ua=1

7. von Groote-Bidlingmaier F, Patientia R, Sanchez E, et al. Efficacy and safety of delamanid in combination with an optimised background regimen for treatment of multidrug-resistant tuberculosis: a multicentre, randomised, double-blind, placebo-controlled, parallel group phase 3 trial. Lancet Respir Med 2019;7:249-59.

8. Pontali E, Centis R, D’Ambrosio L, et al. Recent evidence on delamanid use for rifampicin-resistant tuberculosis. J Thorac Dis 2019;11:S457-60.

9. Mohr E, Hughes J, Reuter A, et al. Delamanid for rifampicinresistant tuberculosis: a retrospective study from South Africa. Eur Respir J 2018;51:1800017.

10. Seung KJ, Khan P, Franke MF, et al. Culture conversion at six months in patients receiving delamanid-containing regimens for the treatment of multidrug-resistant tuberculosis. Clin Infect Dis 2020;71:415-8.

11. Mok J, Kang H, Koh W-J, et al. Final treatment outcomes of delamanid-containing regimens in patients with MDR-/XDRTB in South Korea. Eur Resp J 2019;54:1900811.

12. Olayanju O, Esmail A, Limberis J, et al. A regimen containing bedaquiline and delamanid compared to bedaquiline in patients with drug resistant tuberculosis. Eur Respir J 2020;55:1901181.

13. Mohr E, Ferlazzo G, Hewison C, et al. Bedaquiline and delamanid in combination for treatment of drug-resistant tuberculosis. Lancet Infect Dis 2019;19:470.

14. Pontali E, Sotgiu G, Tiberi S, et al. Combined treatment of drug-resistant tuberculosis with bedaquiline and delamanid: a systematic review. Eur Respir J 2018;52:1800934.

15. Gupta R, Geiter LJ, Hafkin J, et al. Delamanid and QT prolongation in the treatment of multidrug-resistant tuberculosis. Tuberc Lung Dis 2015;19:1261-2.

16. Ferlazzo G, Mohr E, Laxmeshwar C, et al. Early safety and efficacy of the combination of bedaquiline and delamanid for the treatment of patients with drug-resistant tuberculosis in Armenia, India, and South Africa: a retrospective cohort study. Lancet Infect Dis 2018;18:536-44.

17. Borisov S, Danila E, Maryandyshev A, et al. Surveillance of adverse events in the treatment of drug-resistant tuberculosis: first global report. Eur Respir J 2019;54:1901522.

18. Harausz E, Cox H, Rich M, et al. QTc prolongation and treatment of multidrug-resistant tuberculosis. Int J Tuberc Lung Dis 2015;19:385-91.

19. Szumowsk JD, Lynch JB. Profile of delamanid for the treatment of multidrug-resistant tuberculosis. Drug Des Devel Ther 2015;9:677-82.

20. Brigden G, Nhung NV, Skrahina A, et al. Advances in clinical trial design for development of new tb treatments-translating international tuberculosis treatment guidelines into national strategic plans: experiences from Belarus, South Africa, and Vietnam. PLoS Med 2019;16:e1002896.

21. Ministry of Health of The Republic of Belarus [Internet]. Clinical protocol for diagnosis and treatment of tuberculosis among adults and children. 2019. Accessed on: 2019 Nov 20. Available from: http://www.rnpcpf.by/ru/organizacionnometodicheskaya-rabota/normativno-pravovye-akty-po-slujbe.html?start $=15$

22. International Council for Harmonisation of Technical Requirements for Pharmaceuticals for Human Use (ICH) [Internet]. Medical dictionary for regulatory activities (MEDDRA). 2019. Accessed on: 2019 Nov 23. Available from: https://www.meddra.org/

23. US Department of Health and Human Services, National Institutes of Health, National Cancer Institute [Internet]. Common terminology criteria for adverse events (CTCAE) version 5.0. 2017. Accessed on: 2019 Nov 23. Available from: https://ctep.cancer.gov/protocoldevelopment/electronic_applications/docs/CTCAE_v5_Quick_Reference_8.5x11.pdf

24. European Medicines Agency. ICH E2D post-approval safety data management. 1998.

25. Kempker RR, Mikiashvili L, Zhao Y, et al. Clinical outcomes among patients with drug-resistant tuberculosis receiving bedaquiline or delamanid containing regimens. Clin Infect Dis 2019. doi: $10.1093 / \mathrm{cid} / \mathrm{ciz} 1107$ 